\title{
Will conventional cancer chemotherapy soon become obsolete?
}

\section{La chimiothérapie conventionnelle deviendra-t-elle bientôt obsolète ?}

\author{
N.A. Othieno-Abinya \\ (C) Springer-Verlag France 2012
}

Louis Goodman and Alfred Gilman made medical history by inducing regression of non-Hodgkin's lymphoma by administering mustine hydrochloride to patients in 1942. It became apparent for the first time that cancer could be treated with a pharmacologic agent [1]. In the late 1940s and early 1950s, nitrogen mustard alone or combined with glucocorticoids induced significant responses in lymphoid neoplasms.

By early 1960s, cure of acute leukaemias had become a reality with the introduction of antimetabolites including methotrexate, 6-mercaptopurine and cytosine arabinoside. The development of vinca alkaloids soon followed, and this expanded cancer chemotherapeutic armamentarium.

The isolation of daunorubicin in 1961 by A Di Marco at Adria Laboratories in Milan [2] was yet another breakthrough that improved on the cures of acute leukaemia. This was followed in the late 1960s by the isolation of doxorubicin by the same group. Anthracyclines were oncological breakthroughs, with overall single agent response rates exceeding $40 \%$ in treatment-naive malignant lymphomas and acute leukaemia. Impressive response rates were also registered in ovarian and breast cancers. These agents also had major impact in treatment of myeloma, sarcoma, ovarian cancer, non-small cell lung cancer and others.

Notable other chemotherapy successes included the development of epipodophyllotoxins, platinum compounds and taxanes. Cis-platin, carboplatin and etoposide are key agents in treatment of testicular cancer, with the majority of patients cured. The platinum compounds are also effective in treatment of cancers of breast, ovary, lung, bladder, stomach and liver.

Taxanes were first recognised as important chemotherapeutic agents in the 1960s but were only put to clinical use in the early 1990s. Today paclitaxel and docetaxel are invaluable in treatment of cancers of breast, ovary, lung, bladder and others.

\footnotetext{
N.A. Othieno-Abinya $(\triangle)$

Department of Clinical Medicine and Therapeutics,

School of Medicine, College of Health Sciences,

University of Nairobi, Nairobi, Kenya

e-mail : naoabinya@yahoo.com
}

\section{Targeted cancer therapy}

Targeted cancer therapy dates back to the 1970s when the selective oestrogen receptor modifier, tamoxifen, showed efficacy in breast cancer treatment. The use of all-trans-retinoic acid (ATRA) in acute promyelocytic leukaemia, and trastuzumab in Her2-overexpressing breast cancer, were notable targeted therapies of the 1990s.

In the past decade, small molecule tyrosine kinase inhibitors (TKIs) have taken an increasingly important role in the treatment of cancer. These agents were developed to block intracellular signalling pathways in tumour cells, leading to deregulation of key cell functions such as proliferation and differentiation.

The excitement that greeted the introduction of imatinib, an inhibitor of BCR/ABL TK in chronic myeloid leukaemia therapy, left no doubt as to what therapeutic breakthroughs are all about. Nearly all patients with CML in chronic phase achieve complete haematologic remission with this agent, and $80-90 \%$ complete cytogenetic remission [3]. Newer TKIs with even better activity in CML, dasatinib and nilotinib have since been developed.

The successes with imatinib emboldened researchers to concentrate efforts in development of targeted compounds, namely small molecule TKIs, monoclonal antibodies and miscellaneous compounds. Oncologists have been most enthusiastic about these developments for the reasons that: (a) conventional cytotoxics had reached their peak and were not curing any more cancers; and (b) that they were also crude, unselective and toxic. Good luck. It is only in CML where BCR-ABL, targeted by Imatinib, nilotinib and dasatinib, is a single oncogenic driving mutation. Many other efforts have largely been therapeutic misadventures laced with extremely high costs. Their therapeutic activities have been modest to say the least, mainly in combination with chemotherapy.

To be fair, other successes have been exemplified by antiHer2 therapies in combination with chemotherapy, yielding better results than single anti-Her2 agents in breast cancer [4-6]. Trastuzumab, panitumumab and matuzumab are 
notable examples, and also the anti-Her2 small molecule TKI, lapatinib. In NSABP B31 trial by Romond and colleagues, trastuzumab in combination with chemotherapy extended 3-year DFS by $87.1 \%$ compared with $75.4 \%$ for chemotherapy-only arm among patients with operable Her2-positive breast cancer. The overall survival was $94.3 \%$ compared with $91.7 \%$ for chemotherapy only-arm, and the differences were statistically significant [7].

Rituximab: The anti-CD20 monoclonal antibody, rituximab, is another success story in treatment of B-cell lymphoproliferative neoplasms, especially follicular lymphoma and diffuse large B-cell lymphoma [8,9]. The benefits have been clearly demonstrated by various studies including those by Gordon and colleagues, Hainsworth and colleagues and Witzig and colleagues [10-12].

\section{Anti-VEGF therapy}

Treatment directed against vascular endothelial growth factor receptor (VEGFR) has had mixed results. Bevacizumab, the first VEGFR-directed therapy, has been tried in several tumour types with mixed outcomes. For example, addition of bevacizumab to docetaxel and prednisone did not improve overall survival in metastatic castrate-resistant prostate cancer and was associated instead with greater toxicity [13]. Results in colorectal carcinoma have been more encouraging [14].

\section{Newer cytotoxics}

Larotaxel, a newer semisynthetic taxane from the natural compound 10-deacetyl baccatin III, extracted from the tree, Taxus baccata, is showing extremely encouraging results according to a study carried out by Dieras and colleagues [15]. Others are ixabepilone, an epithelone; vinflimine, a vinca alkaloid; eribulin mesylate and the alkylating agent trabectidin. Studies in women with metastatic breast cancer, who have been heavily pretreated, have demonstrated remarkable results with these agents [16-18]. Trabectedin has also shown great promise in sarcomas.

\section{Toxicity profile}

Rash, diarrhoea, skin ulcers, pleural effusions and cardiotoxicity are toxicities being experienced with targeted therapies, while many agents still retain myelotoxicity, mucositis and other side effects that bedevil conventional cytotoxic agents.

Africa, despite having vast natural resources, is the most resource-challenged continent, and patients here can hardly afford the targeted drugs. Whereas a new drug developer is under no obligation to make drugs affordable to Africa and other low-income parts of the world, it would be prudent to justify drug costs on treatment outcome and safety parameters rather than on how much cost in research spending has been accrued even if the agent has no more than placebo effect.

\section{Conclusion}

With the foregoing, it is the contention of the author that conventional cancer chemotherapy is highly unlikely to become extinct soon.

\section{References}

1. Louis SG, Maxwell M, Wintrobe W, et al (1946) Nitrogen mustard therapy: Use of methyl-bis(beta-chloroethyl)amine hydrochloride for Hodgkin's disease, lymphosarcoma, leukaemia, and certain allied and miscellaneous disorders. JAMA 251:2255-61

2. Di Marco A, Cassinelli G, Arcamone F (1981) The discovery of daunorubicin. Cancer Treat Rep 65 (Suppl 4):3-8

3. Druker BJ, Guilhot F, O’Brien SG, et al (2006) Five-year followup of patients receiving imatinib for chronic myeloid leukaemia. N Engl J Med 355:2408-17

4. Baselga J, Bradbury I, Eidtman H, et al (2010) First results of the NeoALTTO Trial (BIG 01-06/EGF 106903): A phase III randomized, open label, neoadjuvant study of lapatinib, trastuzumab and their combination plus paclitaxel in women with HER2-positive primary breast cancer. Cancer Res 70:82s, (abstr s3-3)

5. Gianni L, Pienkowski T, Im YH, et al (2010) Neoadjuvant pertuzumab $(\mathrm{P})$ and trastuzumab $(\mathrm{H})$ : Antitumour and safety analysis of a randomized phase II study ('Neo Sphere'). Cancer Res 70:82s, (abstr s3-2)

6. Untch M, Loibl S, Bischoff J, et al (2010) Lapatinib versus trastuzumab in combination with neoadjuvant anthracyclinetaxane-based chemotherapy: Primary efficacy endpoint analysis of the GEPARQUINTO study (GBG 44). Cancer Res 70:81s, (abstr)

7. Romond EH, Perez EA, Bryant J, et al (2005) Trastuzumab plus chemotherapy for operable HER2-positive breast cancer. N Eng J Med 353:1659-72

8. Herbst RS, Prager D, Hermann R, et al (2005) Phase III trial of erlotinib hydrochloride combined with carboplatin and paclitaxel chemotherapy in advanced NSCLC. J Clin Oncol 23:5892-99

9. Han J, Park K, Kim S, et al (2012) First-SIGNAL: First-line single agent iressa versus gemcitabine and cis-platin trial in never-smokers with adenocarcinoma of the lung. J Clin Oncol $10: 1122-8$

10. Gordon LN, Grow WB, Pusateri A, et al (2005) Treatment for CD20 positive lymphoproliferative disorders. J Clin Oncol 23:1096-102

11. Witzig TE, Vukov AM, Habermann TM, et al (2005) Rituximab in newly diagnosed advanced stage follicular lymphoma. J Clin Oncol 23:1103-8

12. Hainsworth JD, Litchy S, Shafer DR, et al (2005) Maximizing therapeutic benefit of rituximab maintenance therapy versus re-treatment at progression in patients with indolent nonHodgkin lymphoma. A randomized phase II trial of the Minnie Pearl Cancer Research Network. J Clin Oncol 23:1088-95 
13. Kelly WK, Halobi S, Carducci M, et al (2012) Randomized double-blind, placebo-controlled phase III trial comparing docetaxel and prednisone with or without bevacizumab in men with metastatic castration-resistant prostate cancer: CALGB 90401. $\mathrm{J}$ Clin Oncol 30:1534-40

14. Kabbinavar FF, Schutz J, McLeod M, et al (2005) Combined analysis of efficacy of the addition of bevacizumab to 5-FU/LV improves survival for patients with metastatic CRC. J Clin Oncol 23:3697-705

15. Dieras V, Limentani S, Romieu G, et al (2008) Study of larotaxel (XRP9881), a novel taxoid, in patients with metastatic breast cancer who previously received taxane-based therapy. Ann Oncol 19:1255-60
16. Thomas E, Tarbernero J, Fornier M, et al (2007) Phase II clinical trial of ixabepilone (BMS247550), an epithelone analog, in patients with taxane-resistant metastatic breast cancer. J Clin Oncol 25:3399-406

17. Cortes J, Vahdat L, Blum JL, et al (2010) Phase II study of the halichondrin B analog eribulin mesylate in patients with locally advanced or metastatic breast cancer previously treated with an anthracycline, a taxane, and capecitabine. J Clin Oncol 28: 3922-8

18. Zelek L, Yovine A, Brain E, et al (2006) A phase II study of yondelis (trabectedin, ET743) as a 24-hour continuous intravenous infusion in pretreated advanced breast cancer. $\mathrm{Br} \mathrm{J}$ Cancer 94:1610-4 